\title{
๖The Effects of Heavy Precipitation on Polarimetric Radio Occultation (PRO) Bending Angle Observations
}

\author{
Kuo-Nung Wang, ${ }^{a}$ Chi O. Ao, ${ }^{a}$ Ramon Padullés, ${ }^{\mathrm{b}}$ Francis J. Turk, ${ }^{\mathrm{a}}$ Manuel de la Torre JuÁrez, ${ }^{\mathrm{a}}$ \\ AND ESTEL CARDELLACH ${ }^{\mathrm{b}}$ \\ ${ }^{\text {a }}$ Jet Propulsion Laboratory, California Institute of Technology, Pasadena, California \\ ${ }^{\mathrm{b}}$ Institute of Space Sciences (ICE-CSIC), Spain Institute of Space Studies of Catalonia (IEEC), Barcelona, Spain
}

(Manuscript received 22 March 2021, in final form 21 July 2021)

\begin{abstract}
Following the successful launch of the Spanish $P A Z$ mission the proof of concept experiment "Radio Occultation and Heavy Precipitation with $P A Z$ " (ROHP- $P A Z$ ) started operating in May 2018. The ROHP- $P A Z$ observations demonstrated that precise measurements of the phase shift between horizontal and vertical polarizations from Global Navigation Satellite System (GNSS) L-band signals are sensitive to oriented hydrometeors along the ray paths. While this differential phase shift measurement as a function of time has proven very useful, the regular radio occultation (RO) intermediate products from different polarized channels, such as bending angle and phase retrievals on the domain of impact parameter, have never been exploited. In this research, we studied the characteristics of polarimetric phase and bending angle difference retrieved by the radio-holographic $(\mathrm{RH})$ method to mitigate atmospheric multipath effect and to explore their use in data assimilation. To validate RH approach in polarimetric retrievals, we performed end-to-end simulations where the hydrometeors are modeled by the effective refractivity with different horizontal extents. The simulation results demonstrate that the strong precipitation $\left(>15 \mathrm{~mm} \mathrm{~h}^{-1}\right)$ with $40-\mathrm{km}$ horizontal extent can be detected with the retrieved bending angle shift. The calibration process on the impact parameter domain has also been developed to extract the differential phase and bending angle shift from the actual polarimetric RO data. Statistics from the $P A Z$ data shows that the mean retrieved RH polarimetric phase shift with various horizontal extent is approximately proportional to the tangent point location rain rate at a ratio of $0.02 \mathrm{rad}\left(\mathrm{mm} \mathrm{h}^{-1}\right)^{-1}$.
\end{abstract}

KEYWORDS: Global positioning systems (GPS); Measurements; Occultation; Profilers, atmospheric; Remote sensing; Satellite observations

\section{Introduction}

Characterizing the near-surface moisture structure in extreme weather environments remains a challenging problem with current spaceborne remote sensing technology. Passive microwave sounders such as Advanced Technology Microwave Sounder (ATMS) on board Suomi NPP (Kim et al. 2014) only provides the moisture profiles with coarse vertical resolution $(>2 \mathrm{~km})$ and is limited by heavy precipitation. The radar backscatter measurements provided by Precipitation Radar (PR) on the Tropical Rainfall Measuring Mission (TRMM) and DualFrequency Precipitation Radar (DPR) on the Global Precipitation Measurement (GPM) mission cannot distinguish the contribution of water vapor attenuation from the raindrop scattering and causing degraded retrievals in the presence of water vapor. Hyperspectral infrared radiometers such as the Advanced Infrared Sounder (AIRS) do provide the temperature and humidity profile in the lower troposphere but cannot sense through the thick clouds (Wong et al. 2015). On the contrary, Global Navigation Satellite System (GNSS) radio occultation (RO) technique (Kursinski et al. 1997) can provide accurate high-verticalresolution thermodynamic structure observations $(\sim 100 \mathrm{~m})$ with

D Denotes content that is immediately available upon publication as open access.

Corresponding author: Kuo-Nung Wang, kuo-nung.wang@jpl. nasa.gov all-weather operating capability thanks to its L-band wavelength. By tracking the excess signal delay incurred by the curving ray paths due to atmosphere's vertical density gradient, GNSS-RO calculates the bending angle of each ray path and retrieves the refractivity profile of Earth's atmosphere. The resulting refractivity retrievals can then be inverted to acquire the temperature, pressure, and water vapor pressure information, and the retrieved bending angle can be operationally assimilated into numerical weather prediction (NWP) systems to enhance accuracy and range of the forecast.

While the GNSS-RO have been used for observing the thermodynamic structure of convective storms and tropical cyclones in the last decade (Bonafoni et al. 2019), the GNSS signals it utilized do not carry any direct precipitation information as a result of insensitive L-band attenuation to hydrometeors. In May 2018, the Spanish PAZ mission began the proof of concept experiment "Radio Occultation and Heavy Precipitation with $P A Z$ " (ROHP-PAZ) (Cardellach et al. 2014, 2019). The onboard GPS-RO receiver used in this experiment is equipped with dual-polarization capability to receive global positioning system (GPS) signals in separate horizontal $(\mathrm{H})$ and vertical (V) channels. The differential phase shift between the horizontal and vertical components of the electromagnetic field, which can be caused by the asymmetric shape of the falling raindrops along the propagation path due to air drag, can be detected through precise phase measurements in the two polarization channels. The magnitude of the polarimetric phase shift is proportional to the 
amount and size of the hydrometeors, thus providing a unique measurement approach to detect and quantify heavy precipitation. Retaining the benefits from standard GNSS-RO observations, polarimetric RO (PRO) is the first spaceborne remote sensing technique that is able to retrieve high-verticalresolution thermodynamic profiles and precipitation states simultaneously.

Up to now, the PRO concept has only been studied using polarimetric phase shift assuming geometric optics propagation (e.g., analyzed through ray tracing techniques). Cardellach et al. (2014) analyzes the PRO sensitivity using SNR values of COSMIC mission and theoretically predicted that $90 \%$ of the heavy precipitation $\left(>5 \mathrm{~mm} \mathrm{~h}^{-1}\right)$ is detectable with polarimetric phase shift observables. The relationship between the polarimetric phase shift and the heavy rain event is then confirmed in the field campaign results (Padullés et al. 2016a). Some efforts have also been made to retrieve the rain intensity directly from the phase shift along with ray tracing computation. Cardellach et al. (2017) took a probabilistic approach to calculate the most likely precipitation intensity at a given height using lookup table (LUT) based on the observed polarimetric phase shift. Padullés et al. (2016b) untangled the rain intensity and extension using tomography and reconstructed the simple precipitation structure in $2 \mathrm{D}$ occultation plane with mirror-ambiguity respect to the tangent point. It is known, however, that GNSS-RO bending angle observations, rather than the phase measurements, are more commonly used in the NWP data assimilation process. While assessment of individual cases reveals the polarimetric observation uncertainties arising from microphysical and cloud structure effects, the impact of PRO on the NWP process has not yet been assessed. Furthermore, the propagation of the PRO signals is likely to suffer from atmospheric multipath effect due to fine-scale vertical structure of the moisture at lower troposphere, which could introduce additional error when simulated by classical ray tracing algorithms. As a consequence, it may be difficult to take fully advantage of the PRO observations in weather forecasting and science analysis.

In this article we investigated the polarimetric bending angle and phase shift retrieved by phase matching (PM), one of the common radio-holographic ( $\mathrm{RH})$ methods to mitigate atmospheric multipath effect, between $\mathrm{H}$ and $\mathrm{V}$ channels. This new product aims to provide precipitation information through the observed bending angle and phase shift in the impact parameter domain for data assimilation. Once the polarimetric bending angle can be accurately simulated from the known precipitation state, this novel approach can avoid the precipitation retrieval error and the multipath ambiguity introduced by the time domain phase shift assimilation. Here PM is first applied on the PRO signals generated by multiple phase screen (MPS) wave optics (WO) simulation, where a simple forward model is established to calculate the refractivity anomaly due to heavy precipitation with the known rain rate. The details of the polarimetric phase and bending difference simulation and its sensitivity test is described in section 2 . The retrieval process is then applied to the cases from actual $P A Z$ data. In section 3 , the calibration process on impact

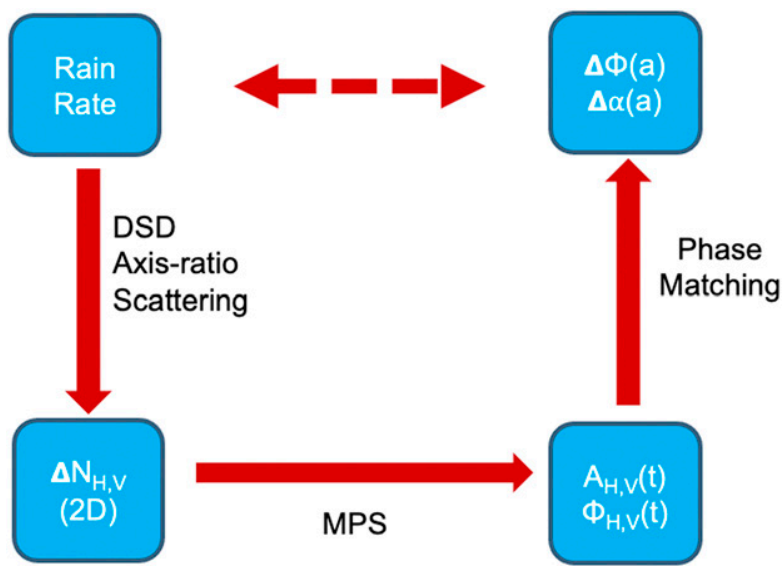

FIG. 1. The flowchart of the end-to-end simulation for polarimetric differential phase $(\Delta \Phi)$ and bending $(\Delta \alpha)$ analysis. The simulation consists of three different parts: 1) 2D refractivity change $(\Delta N)$ calculation for horizontal $(\mathrm{H})$ and vertical (V) channels, 2) MPS forward modeling to calculate received signal amplitude $[A(t)]$ and phase $[\Phi(t)]$ on the time domain, and 3) bending angle and phase retrieval on the impact parameter domain using phase matching.

parameter domain to retrieve polarimetric bending and phase shift from PM is demonstrated. The retrieved polarimetric bending angle and phase shift with different rain rates were compared using the collocated Integrated Multisatellite Retrievals for GPM (IMERG) dataset (Tan et al. 2019). The statistical results of all the available $P A Z$ cases from 10 May 2018 to 7 December 2019 are shown, and the comparison in several case studies that collocated with GPM DPR measurements are also presented. The strengths, limitations, and conclusions of this new PRO product will be further discussed in section 4.

\section{Forward simulation and polarimetric bending angle calculation}

MPS is well-known approach to simulate how radio signals propagate in the known atmosphere. Unlike ray tracing, MPS solves the full wave equation under the parabolic approximation and realistically simulates amplitude and phase observables. In this section, we follow the MPS procedure presented in Beyerle et al. (2002) to construct the end-to-end simulation as shown in Fig. 1 for polarimetric RO observation analysis.

The GNSS signal is assumed to be a plane wave before propagating to Earth's atmosphere and the first screen locates at $x=-1000 \mathrm{~km}$ where the origin locate at the surface of Earth. The receiver is located at $x=1000 \mathrm{~km}$ and moving from $y=60$ to $-250 \mathrm{~km}$ at the speed of $-2.7 \mathrm{~m} \mathrm{~s}^{-1}$. For simplicity, an exponential refractivity structure with the scale height of $7.5 \mathrm{~km}$ and surface refractivity of $300 \mathrm{~N}$ unit is assumed. The $2 \mathrm{D}$ refractivity field along the occultation plane has the vertical resolution of 0.2 and $1 \mathrm{~km}$ for horizontal. A schematic of the MPS on the $2 \mathrm{D}$ radio occultation domain is provided in Fig. 2. To take heavy precipitation into account, the effective refractivity change on different polarizations has 


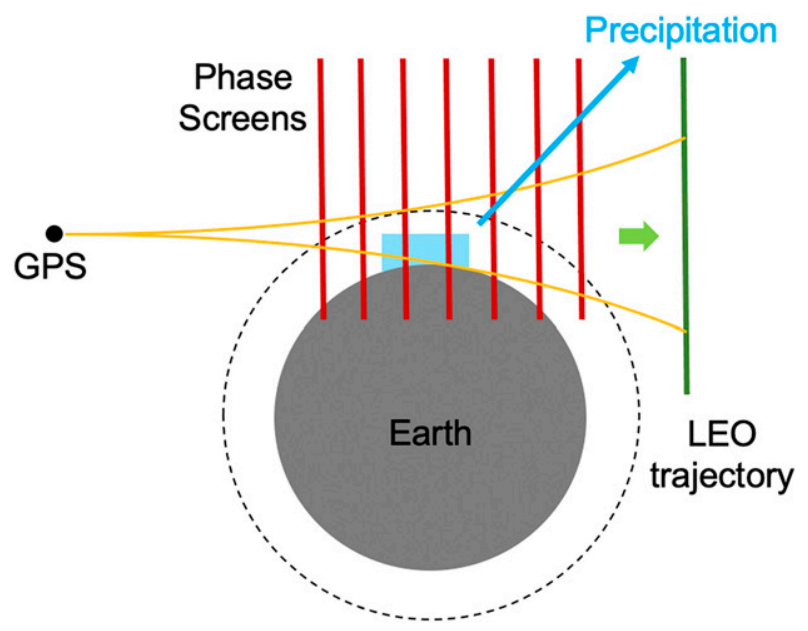

FIG. 2. The GNSS signals propagating through a precipitating 2D domain (blue background) is simulated by a series of phase screens (red lines) depicted in the figure. Each phase screen extends vertically from -250 to $60 \mathrm{~km}$ w.r.t. the surface with $1-\mathrm{km}$ horizontal interval in between. The last screen projected to the simulated LEO trajectory (green line) can be used to derive the amplitude and phase of the received RO signals.

to be calculated before MPS propagation. The effect caused by strong precipitations can then be simulated through retrieving the polarimetric bending angle and phase shift from MPS output using the radio-holographic method. The details of each step in this forward simulation are described below.

\section{a. Refractivity variation due to heavy precipitation}

The heavy precipitation occurs on the occultation plane can be described by the rain rate $\left(\mathrm{mm} \mathrm{h}^{-1}\right)$ for each grid. In this study the probability density of the drop size is modeled by an exponential distribution from Marshall and Palmer (1948), which is a known simplification for any given rain rate:

$$
N_{D}=8000 \exp \left(-4.1 R^{-0.21} D\right),
$$

where $N_{D}$ is the particle concentration $\left(\mathrm{mm}^{-1} \mathrm{~m}^{-3}\right), R$ is the given rain rate $\left(\mathrm{mm} \mathrm{h}^{-1}\right)$ and $D$ is the diameter of the rain drops $(\mathrm{mm})$. The particle concentration $N_{D}$ is a density function where $N_{D} \delta D$ is the number of drops of diameter between $D$ and $D+\delta D$.

For each drop size, its corresponding drop shape can be derived numerically based on the hydrostatic balance between the external aerodynamic pressure and the internal pressure (Beard and Chuang 1987). This model can be approximated by polynomials as shown in Padullés (2017) and Bringi and Chandrasekar (2001) in terms of droplet axis ratio $A_{R}$ :

$$
\begin{aligned}
A_{R}= & 1.0048+5.7 \times 10^{-4} D-2.628 \times 10^{-2} D^{2}+3.682 \\
& \times 10^{-3} D^{3}-1.677 \times 10^{-4} D^{4} .
\end{aligned}
$$

The axis ratio $A_{R}$ is defined as the ratio of vertical axis to the horizontal axis. Note that the calculated $A_{R}$ could exceed 1 in Eq. (2) when the diameter $D$ is closed to zero. In this case we would assume the droplet is a sphere and $A_{R}=1$. In the other cases the droplet shape can be approximated as the oblate spheroid whose semimajor axis lengths are $a, b$, and $c$. Two orthogonal axes along the horizontal directions are equal $(a=b=D / 2)$, and the eccentricity of the oblate spheroid can be derived as

$$
e=\sqrt{1-\frac{c^{2}}{a^{2}}}=\sqrt{1-\frac{\left[(D / 2) A_{R}\right]^{2}}{(D / 2)^{2}}}
$$

The geometrical factor $L_{a}, L_{b}$, and $L_{c}$ of the droplet corresponding to the semimajor axis lengths $a, b$, and $c$ can therefore be calculated (Bohren and Huffman 1983):

$$
\begin{aligned}
& L_{a}=L_{b}=\frac{g}{2 e^{2}}\left[\frac{\pi}{2}-a \tan (g)\right]-\frac{g^{2}}{2}, \\
& L_{c}=1-L_{a}-L_{b},
\end{aligned}
$$

where

$$
g=\sqrt{\frac{1-e^{2}}{e^{2}}} .
$$

As shown in Bohren and Huffman (1983), the horizontal $\left(P_{h}\right)$ and vertical $\left(P_{v}\right)$ polarized electromagnetic phase shift due to scattering by a single oblate spheroid particle can be described as

$$
\begin{aligned}
& P_{h}=4 \pi k a \operatorname{Re}\left[\frac{m^{2}-1}{3+3 L_{a}\left(m^{2}-1\right)}\right], \\
& P_{v}=4 \pi k a \operatorname{Re}\left[\frac{m^{2}-1}{3+3 L_{c}\left(m^{2}-1\right)}\right],
\end{aligned}
$$

where $m=9.3-0.73 j$ is the refractive indices of water at 1.5 $\mathrm{GHz}$ frequency band, $k$ is the GNSS signal wavenumber, and Re is the real part of the complex number. The unit of $P_{h}$ and $P_{v}$ is radians. Consequently, the phase shift of the electromagnetic fields that propagating through a slab of hydrometeors with the thickness of $L$ can be approximated (Gresh 1990):

$$
\begin{gathered}
\Delta \Phi_{h}=\pi a c N_{D} L P_{h}, \\
\Delta \Phi_{v}=\pi a c N_{D} L P_{v} .
\end{gathered}
$$

The phase shift can be related to the refractivity change $\Delta N$ in the GNSS-RO retrieval with given particle size (Zou et al. 2012):

$$
\begin{aligned}
& \Delta N_{h}(D)=\frac{1}{2 \pi k L} \Delta \Phi_{h}, \\
& \Delta N_{v}(D)=\frac{1}{2 \pi k L} \Delta \Phi_{v} .
\end{aligned}
$$

Therefore, the total refractivity shift in a specific grid can be derived by integrating the refractivity change through different drop sizes: 


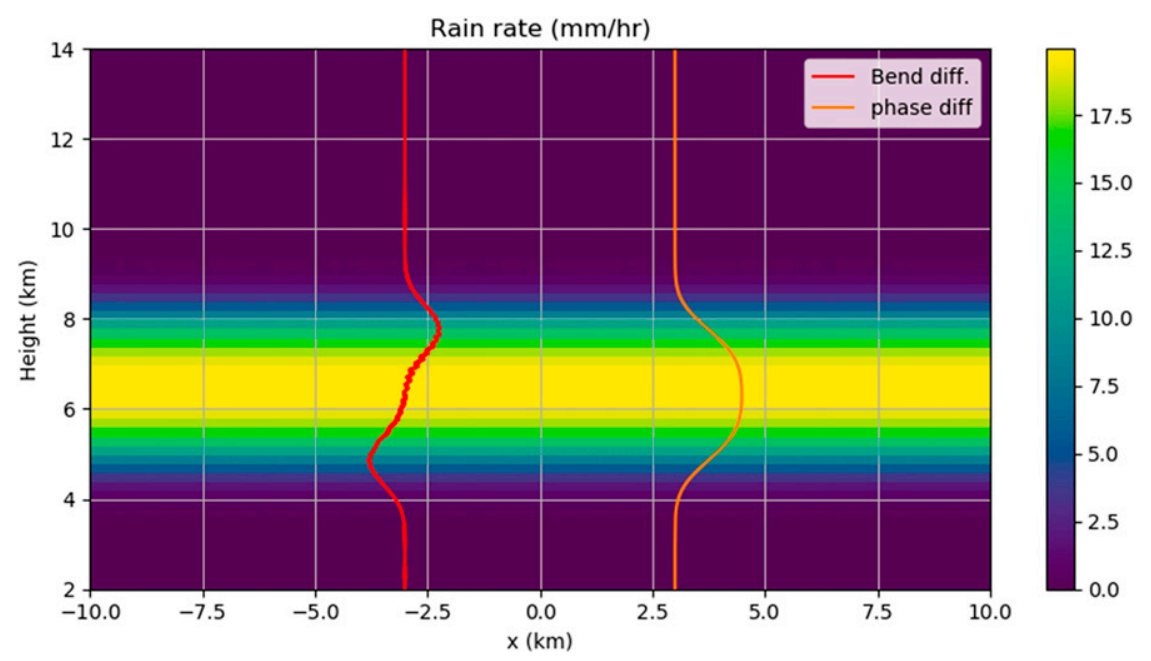

FIG. 3. The simulated bending angle (red) and phase (orange) shift due to hydrometeors overlay with the specified $2 \mathrm{D}$ rain rate (background). The red curve and the orange curve are shifted to $x=-3$ and $3 \mathrm{~km}$, respectively. The results show that the signal phase in the impact parameter domain shifts positively when the heavy precipitation presents, while the bending angle shifts with the strength gradient along the vertical direction.

$$
\begin{aligned}
\Delta N_{h} & =\int \Delta N_{h}(D) d D \\
& =\int 2 \pi N_{D}\left(\frac{D}{2}\right)^{2}\left(\frac{D}{2}\right) A_{R} \operatorname{Re}\left[\frac{m^{2}-1}{3+3 L_{a}\left(m^{2}-1\right)}\right] d D, \\
\Delta N_{v} & =\int \Delta N_{v}(D) d D \\
& =\int 2 \pi N_{D}\left(\frac{D}{2}\right)^{2}\left(\frac{D}{2}\right) A_{R} \operatorname{Re}\left[\frac{m^{2}-1}{3+3 L_{c}\left(m^{2}-1\right)}\right] d D .
\end{aligned}
$$

Through Eqs. (1)-(14), we can calculate the refractivity shift in horizontal and vertical polarizations from the specified rain rate for each grid cell. After the 2D refractivity structure along the occultation plane is constructed for $\mathrm{H}$ and $\mathrm{V}$ we are able to propagate the plane wave signal to both fields independently. The resulting amplitude $A(t)$ and excess phase $\Phi(t)$ for each channel will then be processed with PM algorithm (Jensen et al. 2004) to transform both signals onto the impact parameter domain $A(a) \exp [j \Phi(a)]$ :

$$
A(a) \exp [j \Phi(a)]=\int_{0}^{T} A(t) \exp \left\{j\left[\Phi(t)-\Phi_{o}(a, t)\right]\right\} d t
$$

where the phase matching function $\Phi_{o}(a, t)$ can be described as

$$
\begin{aligned}
\Phi_{o}(a, t)= & k\left\{\sqrt{r_{L}^{2}-a^{2}}+\sqrt{r_{G}^{2}-a^{2}}+a\left[\theta-a \tan \left(\frac{\sqrt{r_{L}^{2}-a^{2}}}{a}\right)\right.\right. \\
& \left.\left.-a \tan \left(\frac{\sqrt{r_{G}^{2}-a^{2}}}{a}\right)\right]\right\} ;
\end{aligned}
$$

$r_{L}$ and $r_{G}$ are the distance from the center of Earth to the LEO satellite and GPS satellite, respectively, and $\theta$ is the open angle between the LEO and GPS satellites. The polarimetric phase shift profile on the impact parameter domain $\Delta \Phi(a)=\Phi_{h}(a)-\Phi_{v}(a)$ and its corresponding bending angle shift $\Delta \alpha(a)$ can be calculated (Jensen et al. 2004):

$$
\Delta \alpha(a) \equiv \alpha_{h}(a)-\alpha_{v}(a)=\frac{1}{k} \frac{d \Delta \Phi(a)}{d a}
$$

where $\alpha_{h}(a)$ and $\alpha_{v}(a)$ are the bending angles of horizontal and vertical polarized signal paths, respectively. Note that the existence of two different bending angles $\left(\alpha_{h}\right.$ and $\left.\alpha_{v}\right)$ does not imply separate ray paths for the two polarization components. Instead, $\alpha_{h}(a)$ and $\alpha_{v}(a)$ should be interpreted as the effective bending angles of the ray paths for transmitted signals with purely $\mathrm{H}$ and $\mathrm{V}$ polarizations, respectively. Since in reality GNSS signals are right-handed circular polarized (RHCP), its actual bending angle $\alpha(a)$ can be computed by combining the $\mathrm{H}$ and $\mathrm{V}$ signals and will lies between $\alpha_{h}(a)$ and $\alpha_{v}(a)$.

\section{b. Results and sensitivity tests}

To illustrate the polarimetric shift in phase and bending angle on the impact parameter domain a simulation example is showed in Fig. 3. An arbitrary but horizontally uniform precipitation structure is specified on a $2 \mathrm{D}$ radio occultation plane where the RO plane waves propagate through. A 2D coordinate is defined as stated in the previous section (Beyerle et al. 2002). The spatial distribution of the precipitation on the grid expanded from 5 to $8 \mathrm{~km}$ in height, and from -20 to $+20 \mathrm{~km}$ horizontally. The rain rate is assumed to be constant $\left(20 \mathrm{~mm} \mathrm{~h}^{-1}\right)$ within the region but the edges (outer 1 $\mathrm{km}$ ) are smoothed so that the rain rate tapers to zero outside the region. This oversimplified precipitation structure may not be realistic but useful for signal propagation analysis and illustration purposes. We overlay the polarimetric phase shift $(x=3 \mathrm{~km})$ and the bending angle shift $(x=-3 \mathrm{~km})$ onto the 
(a)

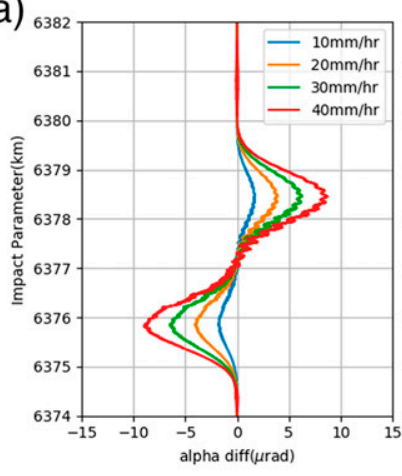

(c)

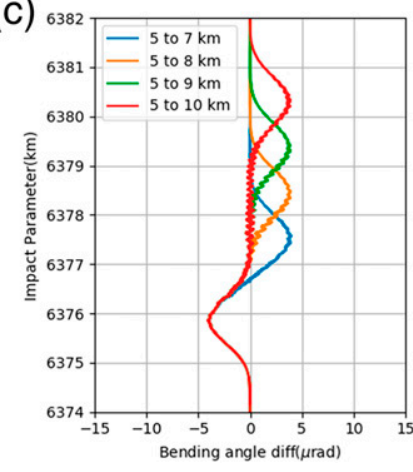

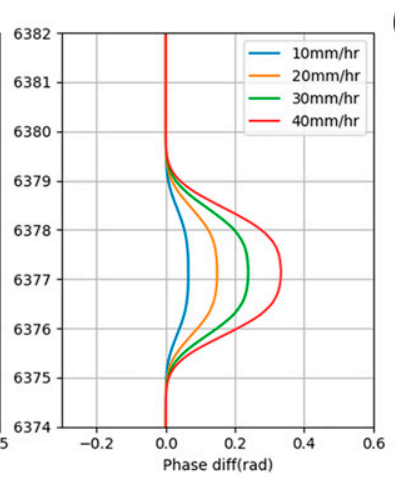

(b)
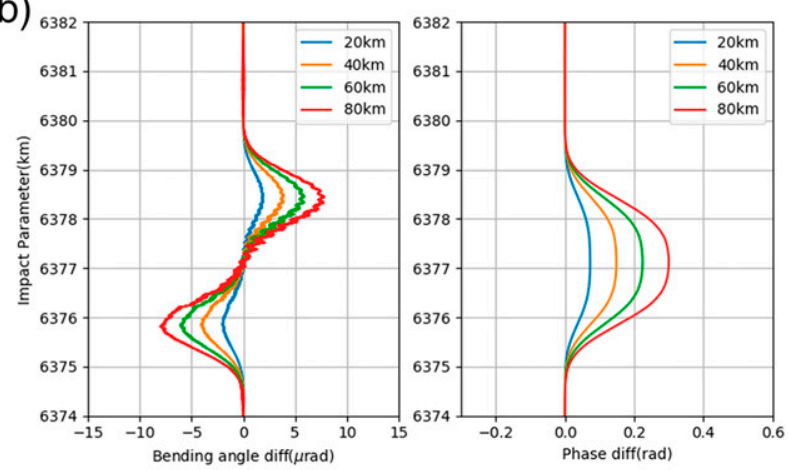

(d)
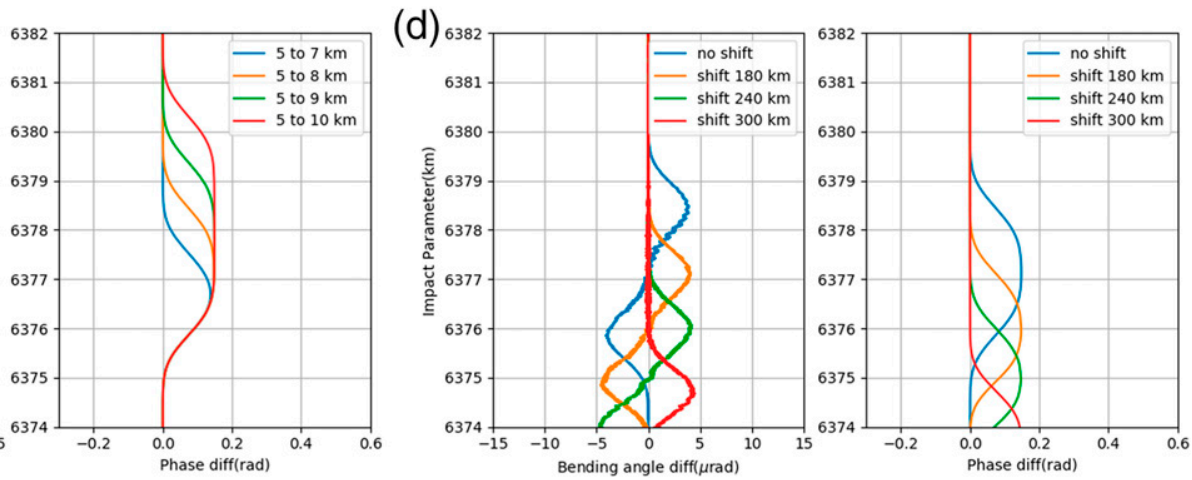

FIG. 4. The sensitivity test of (left) bending angle and (right) signal phase shift on impact parameter domain with respect to (a) precipitation strength (horizontal extent $=40 \mathrm{~km}$, vertical range of precipitation $=5-8 \mathrm{~km}$, horizontal shift in precipitation location $=0 \mathrm{~km}$ ), (b) horizontal extent of precipitation (precipitation strength $=20 \mathrm{~mm} \mathrm{~h}^{-1}$, vertical range of precipitation $=5-8 \mathrm{~km}$, horizontal shift in precipitation location $=0 \mathrm{~km}$ ), (c) vertical range of precipitation (precipitation strength $=20 \mathrm{~mm} \mathrm{~h}^{-1}$, horizontal extent $=40 \mathrm{~km}$, horizontal shift in precipitation location $=0 \mathrm{~km}$ ), and (d) the precipitation location relative to the tangent points (precipitation strength $=20 \mathrm{~mm} \mathrm{~h}^{-1}$, horizontal extent $=40 \mathrm{~km}$, vertical range of precipitation $=5-8 \mathrm{~km}$ ). It is shown that the induced bending angle and phase shift are above

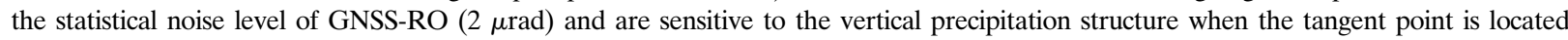
closed enough $(<100 \mathrm{~km})$ to the heavy rain.

2D grid and the results are shown as the orange and red line, respectively, in Fig. 3. As shown in the figure, the polarimetric phase shift (orange line) is positive at precipitation altitude and is vertically related to the specified rain rate. This is mainly due to the ray path is nearly horizontal within the simulated precipitation extent and the rain rate along the ray path is almost constant. On the other hand, the polarimetric bending angle difference (red line) is positive at upper part of precipitation cell while being negative in the lower part of the precipitation. The S-shaped polarimetric bending angle difference structure can be explained by Eq. (17) which is the rate of $\Delta \Phi$, or approximately, the rate of precipitation strength along the vertical axis since the horizontal extent is constant. This simulation shows that the polarimetric phase shift in time domain caused by liquid hydrometeors would be propagated to the impact parameter domain through the radioholographic method, and therefore, the resulting bending angle products from individual channels ( $\mathrm{H}$ and $\mathrm{V}$ ) will also be shifted.

To analyze how polarimetric phase and bending angle shift in impact parameter domain would be induced by different precipitation characteristics, several sensitivity tests based on MPS simulations are conducted. We adjust the strength, horizontal extent, vertical height, and horizontal location shifting of the precipitation given above, and the polarimetric shifting results on impact parameter domain are shown in Fig. 4. The variables used in the test are listed in Table 1. For each subfigure in Fig. 4 the left panel is the bending angle shift and the right panel is the polarimetric phase shift, and different colors corresponds to the levels of precipitation properties. Figure $4 \mathrm{a}$ shows that the magnitude of both shifts is sensitive to the strength of the precipitation, whose peak values are listed in the legends. Noted that the bending angle shift is above the statistical noise level ( $2 \mu \mathrm{rad}$ in actual RO

TABLE 1 . The range of the variables used in the sensitivity test through MPS simulation. Default values are highlighted in boldface fonts. The results are shown in Fig. 4.

\begin{tabular}{cccc}
\hline \hline $\begin{array}{c}\text { Strength } \\
\left(\mathrm{mm} \mathrm{h}^{-1}\right)\end{array}$ & $\begin{array}{c}\text { Horizontal } \\
\text { extent }(\mathrm{km})\end{array}$ & $\begin{array}{c}\text { Vertical } \\
\text { height }(\mathrm{km})\end{array}$ & $\begin{array}{c}\text { Horizontal } \\
\text { location shifting }(\mathrm{km})\end{array}$ \\
\hline 10 & 20 & $5-7$ & $\mathbf{0}$ \\
$\mathbf{2 0}$ & $\mathbf{4 0}$ & $\mathbf{5 - 8}$ & 180 \\
30 & 60 & $5-9$ & 240 \\
40 & 80 & $5-10$ & 300 \\
\hline
\end{tabular}


measurements; Schreiner et al. 2011; Cao et al. 2020) when the precipitation is strong enough $\left(>15 \mathrm{~mm} \mathrm{~h}^{-1}\right)$. However, it can be observed that both phase and bending angle shifts are similar to the cases where the precipitation is fixed and the horizontal extent is adjusted as shown in Fig. 4b. This result verifies the inherent tangling characteristics in PRO measurements between precipitation strength and horizontal extent, as stated in previous works (Padullés et al. 2016b). The vertical range of the precipitation, on the other hand, will not be coupled with the precipitation strength because the ray path closed to the tangent point is nearly horizontal and the vertical structure can be resolved as shown in Fig. 4c. It is worth mentioning that the polarimetric bending angle shift, unlike the polarimetric phase shift, will be zero if the rain rate does not change vertically as the case represented by the red curve in Fig. 4c between 6377 and $6379 \mathrm{~km}$. If the precipitation is located too far from the tangent point horizontally, the ascending shape of the ray path could cause underestimation of the precipitation height as shown in Fig. 4d. In this case it is only noticeable when the precipitation is more than $180 \mathrm{~km}$ away from the tangent point, but the number could depend on the horizontal and vertical extent of the rain cell.

\section{PRO data calibration on impact parameter domain}

While the change in phase and bending on impact parameter domain can be easily observed in the simulation, it is not straightforward to get the phase shift from the actual data. Here we describe the processing of computing the polarimetric phase and bending angle shifts along with the calibration procedure by steps. The calibration process shown here is similar to the ones presented in Padullés et al. (2020) and Cardellach et al. (2019), but here it is applied on the RHretrieved impact parameter domain.

1) $\mathrm{PM}$ processing to transform the received signal from time domain $\left(A_{h}(t) \exp \left[j \Phi_{h}(t)\right], A_{v}(t) \exp \left[j \Phi_{v}(t)\right]\right)$ to impact parameter domain $\left(A_{h}(a) \exp \left[j \Phi_{h}(a)\right], A_{v}(a) \exp \left[j \Phi_{v}(a)\right]\right)$ using Eq. (15).

2) Calculate the phase shift between $H$ and V channels:

$$
\Delta \Phi(a)_{\mathrm{pre}}=\mathrm{UW}\left[\Phi_{h}(a)\right]-\mathrm{UW}\left[\Phi_{v}(a)\right],
$$

where UW is the unwrapping operator. Because the processing of $\mathrm{H}$ and $\mathrm{V}$ channel are independent, the cycle slip could occur if only the unwrapped phase of both channels are differenced. To connect the remaining phase the tan and atan function are applied in the next step.

3) Initialize at $30 \mathrm{~km}$ and connecting the phase shift

$$
\Delta \Phi(a)=a \tan \left\{\tan \left[\Delta \Phi_{\text {pre }}(a)-\Delta \Phi_{\text {pre }}\left(a_{30 \mathrm{~km}}\right)\right]\right\},
$$

where $a_{30 \mathrm{~km}}$ is the impact parameter at the $30-\mathrm{km}$ impact height. We assume the phase shift due to the lack of hydrometeors is zero at this altitude.

4) Remove the linear trend and smooth with low-pass filter. This step is done by fitting a linear function on $\Delta \Phi(a)$ above $10 \mathrm{~km}$ and subtract the extrapolated linear function
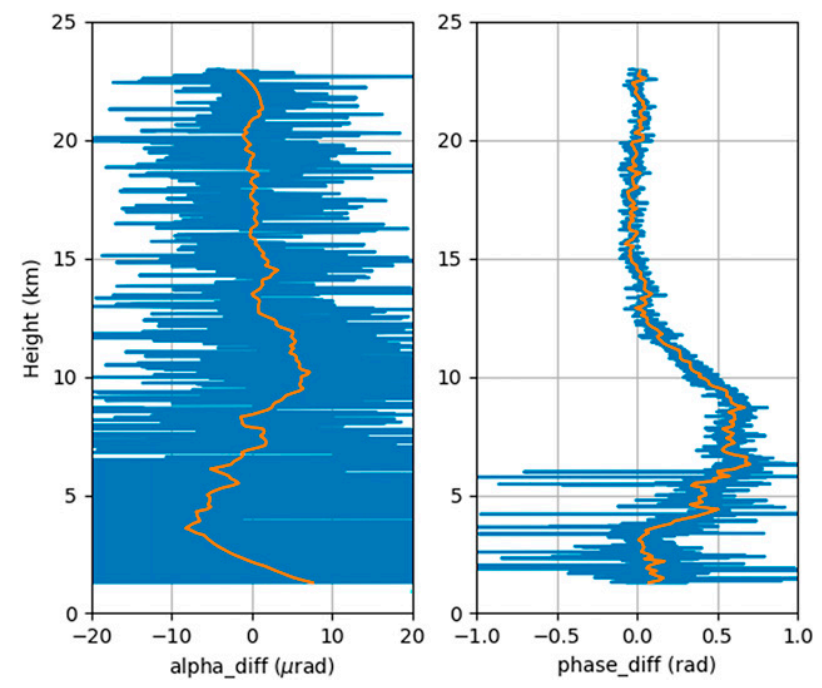

FIG. 5. The (left) bending angle and (right) phase shift between $\mathrm{H}$ and $\mathrm{V}$ channels in a case of actual PAZ data (20180620_ 0658paz_g56, $6.26 \mathrm{~mm} \mathrm{~h}^{-1}$ ). Raw difference is shown in blue curves and the smoothed results are shown in orange curves. The shape of both curves between 4 and $12 \mathrm{~km}$ match the ones derived by MPS simulation.

from $\Delta \Phi(a)$. A 300-m smoothing window low-pass filter is used to extract the polarimetric phase delay.

5) Calculate the bending angle shift $\Delta \alpha(a)$ with Eq. (17).

Note that the antenna effect is not removed in this study, mainly due to the absence of the relationship between time and impact parameter domain in RH methods. The azimuth and elevation angles of the received signals can be a multivalued function of impact parameter $a$. This could cause additional error in the retrieved profiles, and the potential solution is discussed in the next section. An actual $P A Z$ occultation (20180620_0658paz_g56) with $6.26 \mathrm{~mm} \mathrm{~h}^{-1}$ rain rate is used here as an example (Fig. 5). The rain rate of this case is determined from the $0.6^{\circ} \times 0.6^{\circ}$ average of the surface precipitation rain rate provided by the collocated IMERG dataset. The phase and bending angle shift are shown after calibrating the difference of phase on the impact parameter domain. The impact height has been transformed to geometric height for illustration purpose. The blue and orange lines in the right panel are the $\Delta \Phi(a)$ before and after the low-pass smoothing in step 4. An obvious positive phase shift (up to $0.5 \mathrm{rad}$ ) between 4 and $12 \mathrm{~km}$ caused by hydrometeors can be observed. The bending angle shift calculated in step 5 is shown as the blue curve in the left panel of Fig. 5, which is noisy because the fluctuation is amplified through the differentiation. An extra low-pass filter with 3 $\mathrm{km}$ smoothing window is therefore applied to the bending angle difference and the result is shown as the orange curve. The Sshaped profile structure is consistent with the one predicted by MPS simulation. However, without the extra 3-km smoothing the polarimetric phase shift showed in the right panel can better preserve the information in high vertical resolution.

Another actual PAZ case (20181115_0403paz_g53) is shown in Fig. 6 to illustrate the improvement of using PM, 

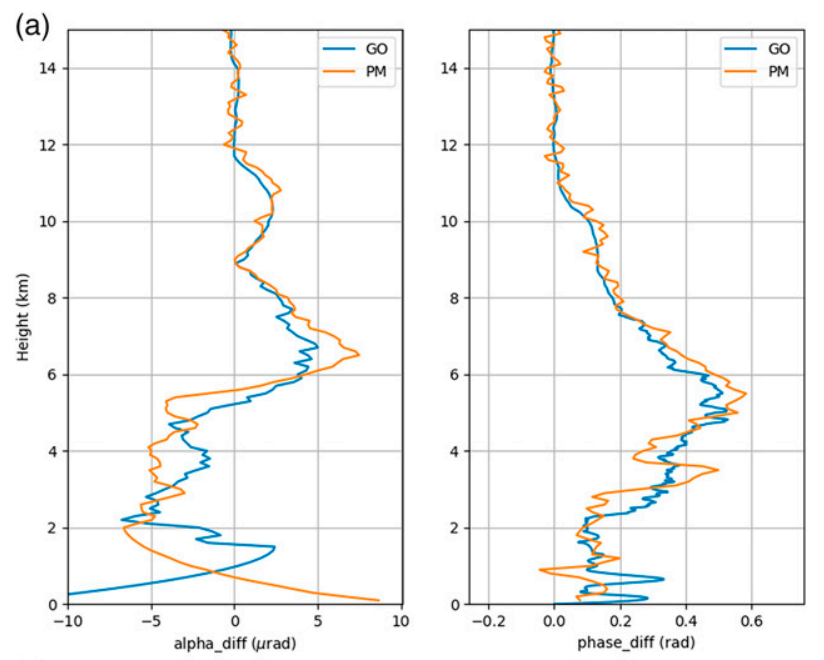

(b)

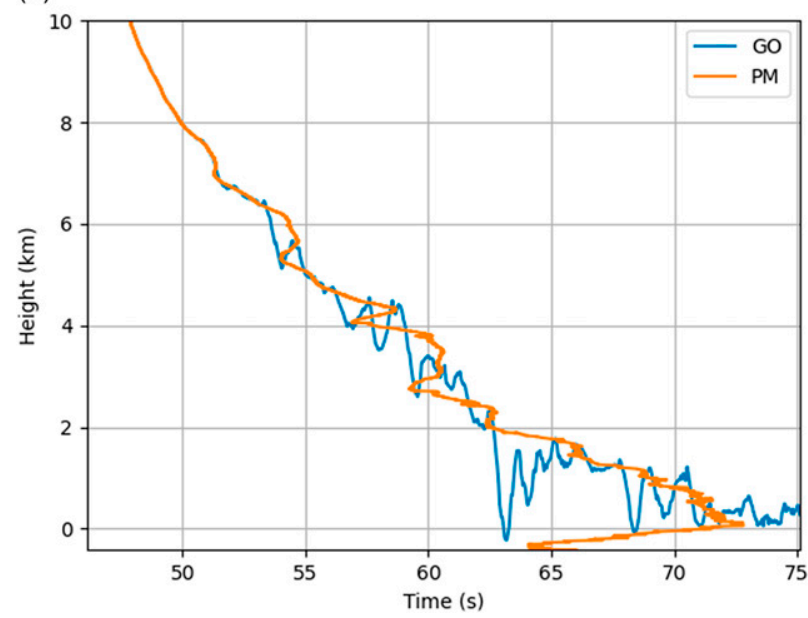

FIG. 6. (a) The (left) bending angle and (right) phase shift between $\mathrm{H}$ and $\mathrm{V}$ channels retrieved by $\mathrm{PM}$ and GO in a case of actual $P A Z$ data (20181115_0403paz_g53, $11.93 \mathrm{~mm} \mathrm{~h}^{-1}$ ). The multipath region between 3 and $4 \mathrm{~km}$ caused the difference between GO and PM, where PM can better resolve the small-scale structure in the phase shift profile. (b) The tangent height as functions of arrival time. Strong fluctuations can be observed in the GO curve when multiple value of tangent height are present in the PM solution. Note that the sharp decrease of arrival time in the PM result below the surface comes from the reflected component of the received signal, which also cause the multipath in GO solution between 63 and $70 \mathrm{~s}$.

one of the radio-holographic methods, over geometric optics (GO) method. In this comparison, the GO polarimetric phase shift is derived by mapping the calibrated phase shift on the time domain (Padullés et al. 2020) to the impact parameter domain based on the impact parameter function $a(t)$ determined by GO. Suffered from multipath effect in the lower troposphere, the impact parameter function $a(t)$ will be nonmonotonic with strong fluctuation when two or more subsignals present. The multivalued $a(t)$ is shown in Fig. 6b and represented by tangent height for illustration purpose. It can be observed that during multipath periods, such as $58-59$ and $\sim 60$ s where multiple tangent heights exist, high level of fluctuation will be introduced in the GO solution and cause erroneous phase shift results between 3 and $5 \mathrm{~km}$. To map the calibrated phase shift on the nonmonotonic impact parameter we sort the impact parameter and its corresponding phase shift, and the results are filtered with the smoothing window of the same length. The phase shift is then differentiated using Eq. (17) to derive the polarimetric bending angle difference. Note that the polarimetric bending angle difference cannot be calculated directly by differencing the bending retrieval from individual channel because the phase difference is not calibrated and the cycle slips between two channels still exist. As shown in Fig. 6a, the polarimetric phase shift (right, blue) retrieved using GO has an overall smoother result because the fine vertical structure in the multipath region has been removed due to the fluctuation caused by impact parameter ambiguity. One apparent difference shows up at height $\sim 3.5 \mathrm{~km}$ where a hump can be observed in the PM profile due to sharp refractivity gradient, while GO is relatively smoother over the region. This is a typical GO-WO difference that can be frequently observed in the bending angle retrieval.

\section{a. Statistical results}

To show the relationship between the polarimetric phase shift and the precipitation level, we chose 525 PAZ occultations between August 2018 and May 2019 for statistical analysis. For each case the collocations are performed with $0.6^{\circ} \times$ $0.6^{\circ}$ averaged IMERG precipitation rain rates to calculate its precipitation strength. These cases are then categorized into 11 groups of different integer precipitation levels: $0-10 \mathrm{~mm} \mathrm{~h}^{-1}$, based on the integer floor of their collocated IMERG rain rate. For example, the cases with the collocated rain rate between 2 and $3 \mathrm{~mm} \mathrm{~h}^{-1}$ are designated as the $2 \mathrm{~mm} \mathrm{~h}^{-1}$ group. As expected, most of the $P A Z$ cases during this 10month period is rain-free or with weak precipitation. To make the sample number comparable, we randomly selected 100 cases for the first three groups $\left(0,1\right.$, and $\left.2 \mathrm{~mm} \mathrm{~h}^{-1}\right)$ and preserve all the cases of the other groups. The statistical results along with the actual case number for each group is showed in Figs. 7 and 8. In Fig. 7, the mean value and standard deviation of bending angle shift (Fig. 7a) and the polarimetric phase shift (Fig. 7b) with respect to height (vertical axis) and rain rate (color) are demonstrated. It can be clearly observed from the statistics that the precipitated cases ( $>5$ and $0-2 \mathrm{~mm}$ ) contain positive mean and standard deviation of polarimetric phase shift, and the value of shift is higher when the precipitation is stronger $(>5 \mathrm{~mm})$. The jump of the standard deviation at $5 \mathrm{~km}$ could be due to the lack of calibration on antenna pattern. The positive phase shift of the precipitated cases extended from the surface to $>12 \mathrm{~km}$, implies the ice particles could also cause depolarization of GNSS signals. Unlike the positive-signed phase shift, the polarimetric bending angle shift is the rate of the phase shift which can be both positive and negative. As a result, the mean bending angle shift is averaged evenly over the altitudes and the results from different groups can be barely distinguished. However, the mean polarimetric bending shift 
(a)

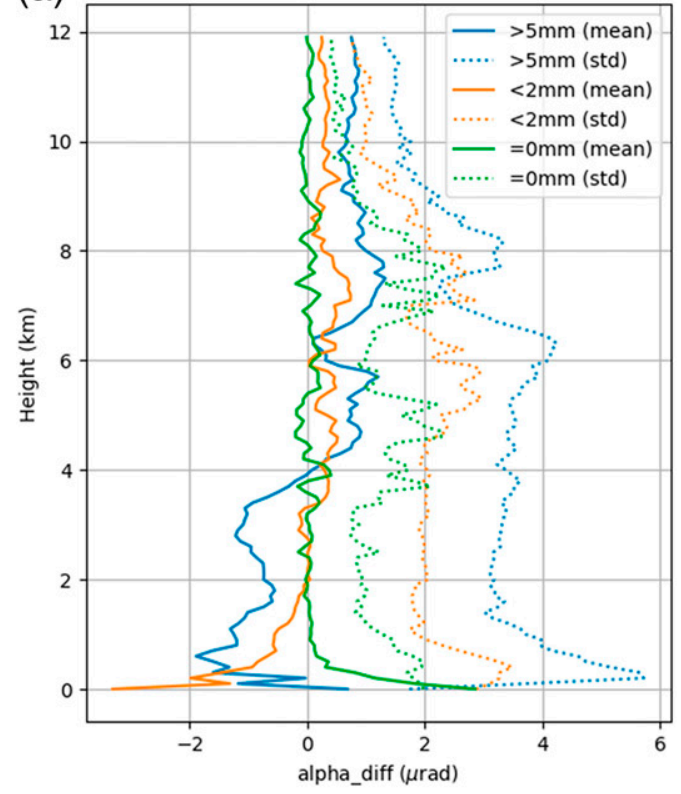

(b)

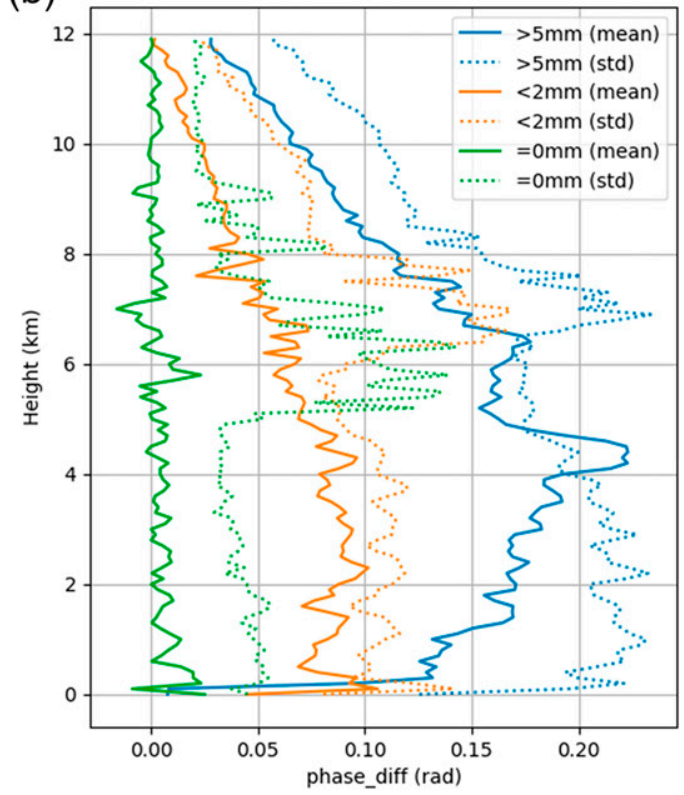

(c)

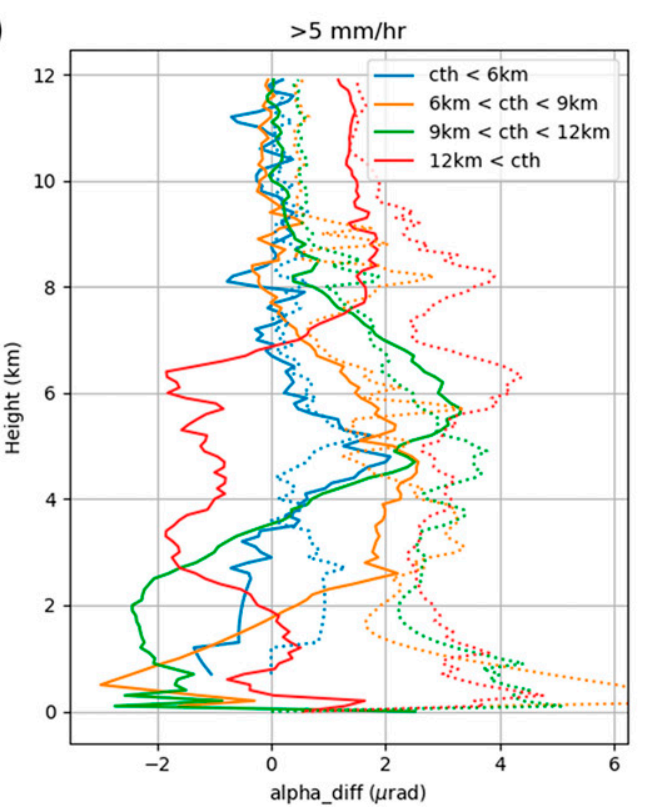

FIG. 7. The (a) bending angle and (b) phase shift between $\mathrm{H}$ and $\mathrm{V}$ channels with respect to heights separating with different collocated precipitation strength (blue: strong rain; orange: weak rain; green: no rain). The solid lines are mean values and the dotted lines are the standard deviation (std) of the shifts. It can be observed that the mean phase shift and the bending angle shift std are sensitive to the strength of the rain. (c) Note that the mean bending angle shift is not sensitive because the positive and negative shifts cancels out in averaging, but the S-shape structure will show when they are grouped by similar CTH.

will show if the cases are further categorized by different cloud-top heights $(\mathrm{CTH})$, which is defined as the height of RO temperature profile that matches the infrared brightness temperature (Cardellach et al. 2019). Figure 7c shows the results of different $\mathrm{CTH}$ in the $>5 \mathrm{~mm} \mathrm{~h}^{-1}$ group, and the S-shaped structure can be observed for each $\mathrm{CTH}$ group because the shift will not be cancelled between similar CTH cases. Here the bending shift sensitivity to the precipitation strength is showed by examining the standard deviation (dotted lines in Fig. 7a) since the magnitude of the bending angle shift, regardless of its sign, depends on the rain rate close to the tangent point location (Fig. 4). Figure 7a shows that the 


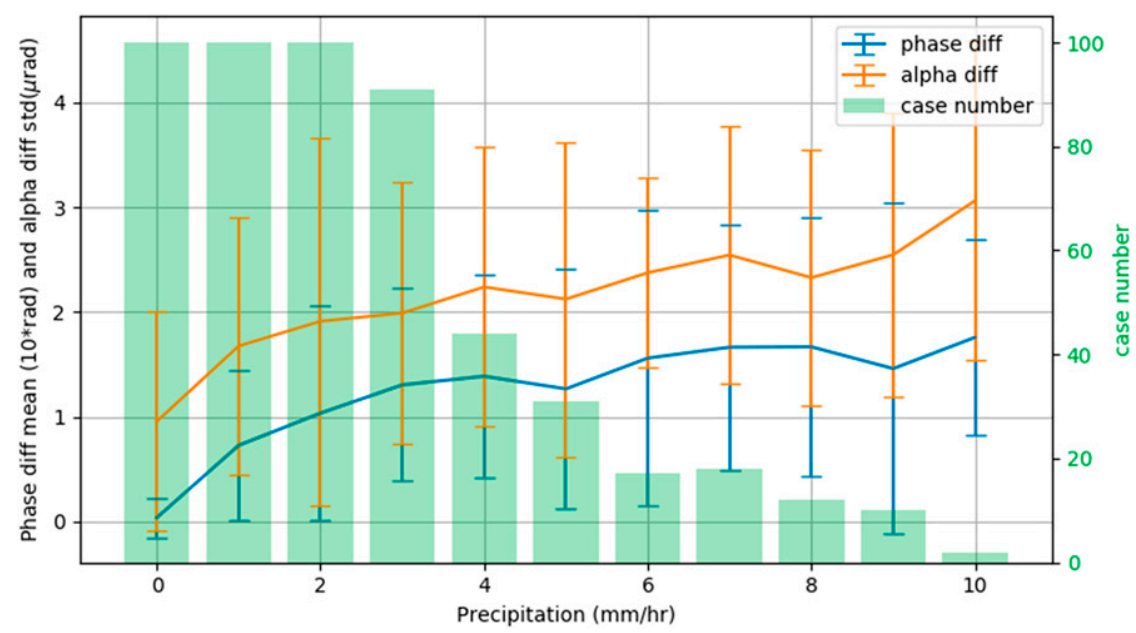

FIG. 8. The average phase shift (blue line) and bending angle difference standard deviation (orange line) with respect to the collocated precipitation strength. The case number for each group is shown in green bars at the background. Both curves show a positive relationship with the rain strength, indicating the phase shift and bending angle standard deviation on impact parameter domain can be used to characterize nearby precipitations.

standard deviation in the bending angle shift does have a positive correlation with respect to the precipitation strength (>3 $\mu \mathrm{rad}$ for strong rain group while only $\sim 1 \mu \mathrm{rad}$ for no rain group).

Figure 8 shows the change of the polarimetric phase shift in impact parameter domain (blue) and differential bending angle standard deviation (orange) as functions of rain rate in details. The phase shift are averaged and the bending standard deviation are calculated over heights from 2 to $8 \mathrm{~km}$ for each case, and the mean phase shift and bending standard deviation shown in Fig. 8 were calculated over all the cases in the same group. Overall, both curves show logarithmic increasing over precipitation levels. The mean polarimetric phase shift increased from nearly 0 rad in no precipitation cases to $0.18 \mathrm{rad}$ for $10 \mathrm{~mm} \mathrm{~h}^{-1}$ cases, while the bending angle shift standard deviation increased from 1 to $3 \mu \mathrm{rad}$ with the same condition. In other words, without the knowledge of horizontal extent distribution, the mean polarimetric phase shift and differential bending angle standard deviation are approximately proportional to the tangent point precipitation strength at a rate of $0.02 \mathrm{rad}\left(\mathrm{mm} \mathrm{h}^{-1}\right)^{-1}$ and $0.2 \mu \mathrm{rad}$, respectively. Lower sensitivity at stronger precipitation levels can be originated from the nonlinear relationship between rain rate and drop axis ratio, different orientation of the ice particles, and various horizontal extents of different cases. The fluctuations of both curves could be caused by the low case numbers (showed as green bars) for high precipitation groups, which can be eased when more cases being included in the future. But in general, both polarimetric phase shift and the bending angle shift are proved to be sensitive to the precipitation strength according to the actual $P A Z$ mission statistics.

The individual cases used for the statistical study are plotted on the global map in Fig. 9 to show the spatial distribution of $P A Z$ observations. The rain rate and the maximum phase shift of each case are represented by the size and color of marker, respectively. The maximum phase shift is used here instead because the mean phase shift of individual cases could be easily influenced by the tangent point drift, which cannot sufficiently represent the precipitation at a single location. It can be observed that most of the heavy precipitation cases $\left(>7 \mathrm{~mm} \mathrm{~h}^{-1}\right)$ occur over the ocean where radiosonde observations are sparse, stresses the importance of using spaceborne instruments for vertical precipitation and thermodynamic structure studies. Typically these stronger precipitation cases have larger maximum phase shift $(>0.2 \mathrm{rad})$, while the weaker precipitation cases (smaller size markers) tend to have smaller maximum phase shift. Some exceptions can be observed in Fig. 9, which could be mainly due to the missing information of horizontal extent and the horizontal RO ray path drifts.

\section{b. Case study}

A single $P A Z$ case study (20180525_0220paz_g57) is provided in this subsection to demonstrate the phase and bending angle shift due to precipitation. This case is chosen because the collocated rain-rate data provided by GPM (Olson 2017) exist. The precipitation product through the combination of GPM Microwave Imager (GMI) and DPR instruments is used, which provides $3 \mathrm{D}$ rain rate in $25-\mathrm{km}$ and $250-\mathrm{m}$ horizontal and vertical resolution, respectively. The collocated GPM and PAZ data are shown in Fig. 10a from the nadir viewing angle. The red dots in Fig. 10a are the $P A Z$ tangent point locations for each ray path, which are shown in black solid lines. Due to the refractivity variation at lower troposphere the tangent point locations are irregular at low altitudes (left side of the tangent point trajectory). The color from background is the total precipitation rate at the surface acquired from the GPM product on the horizontal grid. It can be observed from Fig. 10a that the $P A Z$ signals propagates 


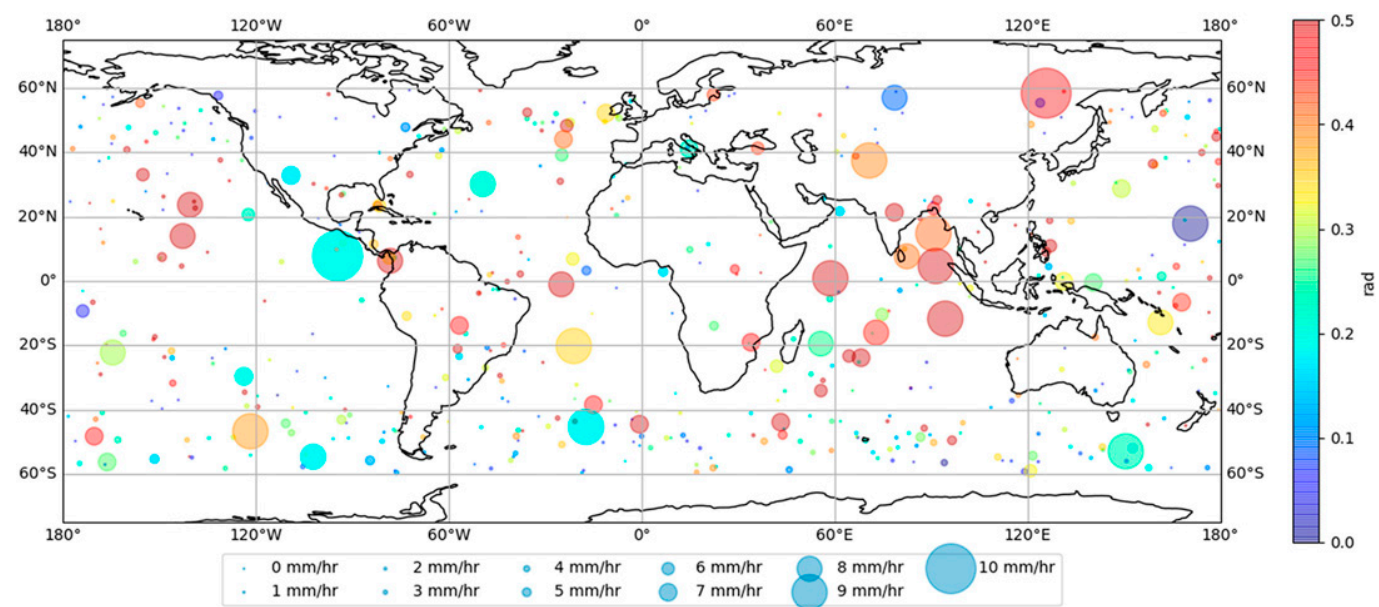

FIG. 9. The spatial scatterplot of 525 cases of $P A Z$ mission chosen for this statistical study between August 2018 and May 2019. The precipitation strength is identified by the size of the marker and its color indicates the corresponding maximum phase shift of the same case. In general, larger maximum phase shift can be detected in higherrain-rate events and vice versa. The case number distribution of each rain-rate group is shown in Fig. 8.

through a heavy precipitation region around $55^{\circ} \mathrm{N}, 58^{\circ} \mathrm{E}$. Note that this case is located at the edge of the GPM swath and some of the rain-rate information along the ray path is missing at lower altitudes.

To compare with the $P A Z$ polarimetric phase and bending angle shift the $3 \mathrm{D}$ rain rate retrieved from GPM is replotted on the 2D occultation plane as shown in Fig. 10b. The tangent point at each height are shifted horizontally in this subfigure to the location $x=0$ and the rain rate between the horizontal range of -100 to $100 \mathrm{~km}$ for each ray path are shown. The heavy precipitation $\left(\sim 10 \mathrm{~mm} \mathrm{~h}^{-1}\right)$ around $2-3 \mathrm{~km}$ height can be observed at $-75 \mathrm{~km}$ with respect to the tangent point location. The small rain rate below $2 \mathrm{~km}$ is due to the $P A Z$ cutoff height $(\sim 1 \mathrm{~km})$ in this case and the horizontal drift of the ray path so that it missed the heavy precipitation region below $2 \mathrm{~km}$. The corresponding polarimetric bending angle (left) and phase shift (right) with respect to height are shown in Fig. 10c. Both panels show a large variation below $6 \mathrm{~km}$ height, while bending angle shift remains neutral and polarimetric phase shift starts biasing positively. At around $2 \mathrm{~km}$ a peak $(0.2 \mathrm{rad})$ in the phase shift is shown, which agrees with the height of the observed precipitation from GPM. Slightly lower peak location could be due to the lower tangent point altitude compared to the precipitation crossed location at the same ray path. The positive phase shift on the impact parameter domain between 3 and $6 \mathrm{~km}$ indicates the polarimetric phase could be sensitive to the ice particles (Padullés et al. 2021). The consistency between polarimetric phase shift result and GPM data shows the possibility of using this information for data assimilation purposes.

\section{Conclusions and discussion}

This article demonstrates that the polarimetric phase and bending angle shift retrieved from the polarimetric RO
(PRO) measurements using PM techniques are sensitive to the heavy precipitation in both simulation and actual cases. The wave-optics retrieval algorithm (phase matching) and the calibration process to extract the phase and bending angle shift from actual data are described in detail. The relationship between the precipitation strength and the polarimetric shift measurements on the impact parameter domain are analytically derived by modeling the refractivity shifts due to asymmetric hydrometeor scattering. By applying the multiple phase screen (MPS) technique to propagate the plane waves through the $2 \mathrm{D}$ refractivity fields the phase and bending angle shift between horizontal (H) and vertical (V) channels can be simulated. A sensitivity study using the MPS simulations illustrates that both measurements are sensitive to the rain rate, precipitation altitude, and the horizontal extent of the rain cell. These relationships are validated by a statistical study from 525 actual $P A Z$ mission cases collocated with IMERG dataset. The results show that the bending angle and phase shift, which can be easily calculated from the existing RO retrieval procedure, are feasible observations not only for the next generation RO data assimilation process that takes precipitation and convection into account, but also benefits the scientific studies such as atmospheric convection process and precipitation climatology (Turk et al. 2021).

While the bending angle and phase shift calculation in the impact parameter domain is straightforward, this algorithm is limited by two factors. First, the PM method is derived under the assumption of spherical symmetric atmosphere. However, this assumption is violated under the precipitation conditions which only limited range of the layer could have its refractivity changed due to scattering. Although the bending accuracy degradation caused by this horizontal refractivity gradient is uncertain, its effect is expected to be not significant since the refractivity change due to precipitation is normally less than $0.2 \mathrm{~N}$ unit, while the uneven water vapor distribution can 
(a)

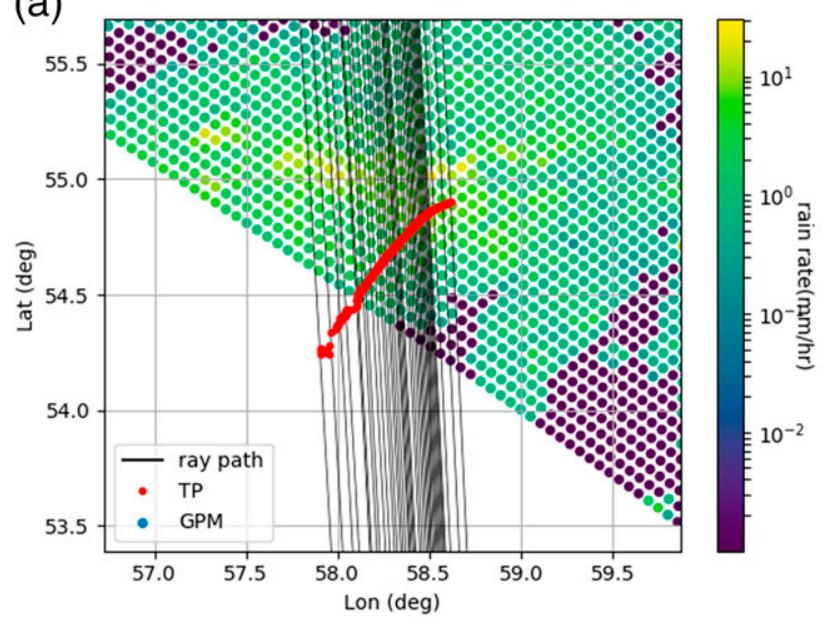

(c)

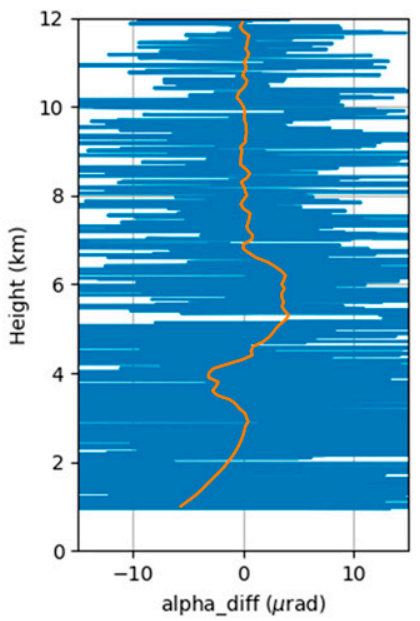

(b)

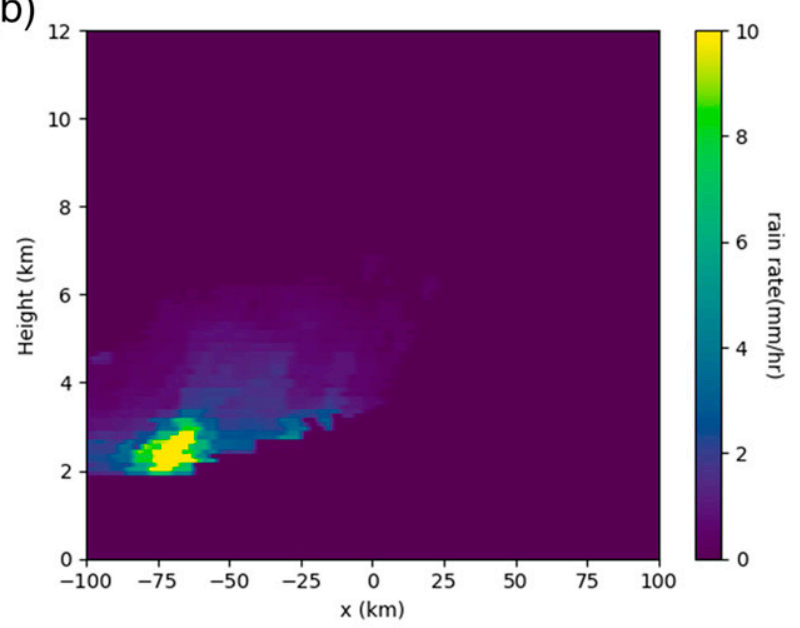

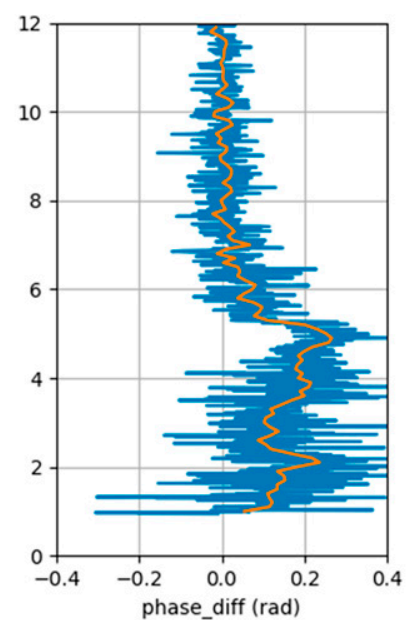

FIG. 10. An actual PAZ case (20180525_0220paz_g57) collocated with GPM to illustrate the polarimetric phase and bending angle shift with respect to the rain rate. (a) The nadir-looking 2D map of PRO and GPM observations. Red dots are the tangent points for each ray paths, and the black lines are the projected ray path on the surface. The colors of the background dots are the surface rain rate retrieved by GPM. (b) The GPM rain rate on the occultation plane. The tangent point location for all paths are shifted to $x=0$. (c) The (left) polarimetric bending angle and (right) phase shift retrieved with PM. Blue curves are the original data, and the orange curves are the vertically smoothed results.

bring much larger horizontal refractivity gradient. Another limitation is the lack of antenna pattern removal in the calibration process. It was done in Padullés et al. (2020) by computing the phase shift of the received signals at each azimuth and elevation angle pairs and connects them to the corresponding time steps. However, the time information is lost in the $\mathrm{RH}$ approach since the signal has been transformed to the impact parameter domain, and the application of this removal method is not feasible. One potential solution is to calculate the subsignal "arrival time" (Jensen et al. 2003) at each impact parameter and interpolate the antenna pattern phase shift based on the derived azimuth and elevation angle corresponding to the specified arrival time. The calculated antenna phase shift bias can then be removed from the polarimetric phase shift retrievals, but this approach needs further investigations.
Currently, the methodology of assimilating the PRO phase shift measurements in the time domain is under development. As typical RO data assimilation process, the geometrical optics approach is used and the signal paths on the known 2D refractivity field at each time step is calculated through the ray tracing algorithm. By integrating the differential phase shift caused by nonspherical hydrometeors along each ray trajectory the total polarimetric phase shift on the time domain will be obtained. The differential phase shift at each point along the ray path can be calculated by combining the hydrometeor scattering model and radiative transfer solver, such as the package provided by Community Radiative Transfer Model (CRTM). This approach has been implemented to the Unified Forward Operator (UFO) in Joint Effort for Data assimilation Integration (JEDI), a coupled data assimilation system developed by Joint Center for Satellite Data 
Assimilation (JCSDA). Alternatively, one can also use the separately calculated 2D refractivity field for $\mathrm{H}$ and $\mathrm{V}$ polarized signals assuming the drop size distribution is known and the hydrometeor axis ratio can be modeled for each drop size as shown in section 2 a. These two refractivity fields will be used to perform ray tracing program twice, and the timedomain phase shift for each field can be obtained. By taking the difference between the phase of different channels one can evaluate the polarimetric phase shift on the time domain from the known precipitation and convection status, and compare them with the ones observed from the data. Since the polarimetric bending angle and phase shift on the impact parameter domain can be retrieved as proposed in this article, potentially they can be directly used through $2 \mathrm{D}$ forward operator in the data assimilation process. However, the ray tracing algorithm derived from geometrical optics could still limit the forward operator under multipath circumstances caused by sharp vertical refractivity changes. To take fully advantage of radio-holographic retrieved bending angle observations a novel forward operator that can propagate the signal using wave optics with acceptable processing time will be ideal, which requires further studies in the future.

Acknowledgments. The research described in this paper was carried out at the Jet Propulsion Laboratory (JPL), California Institute of Technology, under a contract with the National Aeronautics and Space Administration. It is supported by NASA ROSES Earth Science U.S. Participating Investigator Program 14-ESUSPI14-0014. The GNSS PRO experiment aboard $P A Z$ is funded by the Spanish Grant MCI RTI2018-099008-B-C21/AEI/10.13039/501100011033/ERDF. E.C. is member of the EUMETSAT ROM SAF. We would also like to thank Goddard Earth Sciences Data and Information Services Center (GES DISC) for providing GPM level-3 precipitation data and IMERG product.

Data availability statement. The processed $P A Z$ data can be accessed through the following link: https:/genesis.jpl. nasa.gov/ftp/pub/genesis/glevels/paz/.

\section{REFERENCES}

Beard, K. V., and C. Chuang, 1987: A new model for the equilibrium shape of raindrops. J. Atmos. Sci., 44, 1509-1524, https://doi.org/10.1175/1520-0469(1987)044<1509:ANMFTE >2. $0 . \mathrm{CO} ; 2$.

Beyerle, G., K. Hocke, J. Wickert, T. Schmidt, C. Marquardt, and C. Reigber, 2002: GPS radio occultations with CHAMP: A radio holographic analysis of GPS signal propagation in the troposphere and surface reflections. J. Geophys. Res., 107, 4802, https://doi.org/10.1029/2001JD001402.

Bohren, C. F., and D. R. Huffman, 1983: Absorption and Scattering of Light by Small Particles. Wiley-Interscience, 544 pp.

Bonafoni, S., R. Biondi, H. Brenot, and R. Anthes, 2019: Radio occultation and ground-based GNSS products for observing, understanding and predicting extreme events: A review. Atmos. Res., 230, 104624, https://doi.org/10.1016/j.atmosres.2019.104624.

Bringi, V. N., and V. Chandrasekar, 2001: Polarimetric Doppler Weather Radar: Principles and Applications. Cambridge University Press, 380 pp.
Cao, C., W. Wang, E. Lynch, Y. Bai, S. Ho, and B. Zhang, 2020: Simultaneous radio occultation for intersatellite comparison of bending angles toward more accurate atmospheric sounding. J. Atmos. Oceanic Technol., 37, 2307-2320, https://oi.org/10. 1175/JTECH-D-20-0036.1.

Cardellach, E., and Coauthors, 2014: Sensitivity of PAZ LEO polarimetric GNSS radio-occultation experiment to precipitation events. IEEE Trans. Geosci. Remote Sens., 53, 190-206, https://doi.org/10.1109/TGRS.2014.2320309.

— , R. Padullés, S. Tomás, F. J. Turk, C. O. Ao, and M. de la Torre-Juárez, 2017: Probability of intense precipitation from polarimetric GNSS radio occultation observations. Quart. J. Roy. Meteor. Soc., 144 (Suppl. 1), 206-220, https://doi.org/10. 1002/qj.3161.

— S. Oliveras, A. Rius, S. Tomás, C. O. Ao, and G. W. Franklin, 2019: Sensing heavy precipitation with GNSS polarimetric radio occultations. Geophys. Res. Lett., 46, 1024-1031, https://doi.org/ 10.1029/2018GL080412.

Gresh, D. L., 1990: Voyager radio occultation by the Uranian rings: Structure, dynamics, and particle sizes. Ph.D. dissertation, Stanford University, $202 \mathrm{pp}$.

Jensen, A. S., M. S. Lohmann, H.-H. Benzon, and A. S. Nielsen, 2003: Full spectrum inversion of radio occultation signals. Radio Sci., 38, 1040, https://doi.org/10.1029/2002RS002763.

,,-- A. S. Nielsen, and H.-H. Benzon, 2004: Geometrical optics phase matching of radio occultation signals. Radio Sci., 39, RS3009, https://doi.org/10.1029/2003RS002899.

Kim, E., C.-H. J. Lyu, K. Anderson, R. V. Leslie, and W. J. Blackwell, 2014: S-NPP ATMS instrument prelaunch and onorbit performance evaluation. J. Geophys. Res. Atmos., 119, 5653-5670, https://doi.org/10.1002/2013JD020483.

Kursinski, R., G. Hajj, J. Schofield, R. Linfield, and K. Hardy, 1997: Observing Earth's atmosphere with radio occultation measurements using the global positioning system. J. Geophys. Res., 102, 23429-23465, https://doi.org/10.1029/ 97JD01569.

Marshall, J. S., and W. M. Palmer, 1948: The distribution of raindrops with size. J. Meteor., 5, 165-166, https://doi.org/10.1175/ 1520-0469(1948)005<0165:TDORWS>2.0.CO;2.

Olson, W., 1987: GPM DPR and GMI combined precipitation L2B 1.5 hours $5 \mathrm{~km}$, version 06. GES DISC, accessed 6 March 2019, https://doi.org/10.5067/GPM/DPRGMI/ $\mathrm{CMB} / 2 \mathrm{~B} / 06$.

Padullés, R., 2017: Precipitation measurements with polarimetric radio occultations. Ph.D. thesis, Universitat de Barcelona, 246 pp.

— , E. Cardellach, M. de la Torre Juárez, S. Tomás, F. J. Turk, S. Oliveras, and A. Rius, 2016a: Atmospheric polarimetric effects on GNSS radio occultations: The ROHP-PAZ field campaign. Atmos. Chem. Phys., 16, 635-649, https://doi.org/ 10.5194/acp-16-635-2016.

,$--\longrightarrow$, and A. Rius, 2016b: Untangling rain structure from polarimetric GNSS radio occultation observables: A 2D tomographic approach. Eur. J. Remote Sens., 49, 571-585, https://doi.org/10.5721/EuJRS20164930.

— , C. O. Ao, F. J. Turk, M. de la Torre Juárez, B. Iijima, K. N. Wang, and E. Cardellach, 2020: Calibration and validation of the Polarimetric Radio Occultation and Heavy Precipitation experiment aboard the PAZ satellite. Atmos. Meas. Tech., 13, 1299-1313, https://doi.org/10.5194/amt-13-1299-2020.

- E. Cardellach, F. J. Turk, C. O. Ao, M. de la Torre Juárez, J. Gong, and D. L. Wu, 2021: Sensing horizontally oriented frozen particles with polarimetric radio occultations aboard 
PAZ: Validation using GMI coincident observations and CloudSat a-priori information. IEEE Trans. Geosci. Remote Sens., 60, 4101513, https://doi.org/10.1109/TGRS.2021. 3065119.

Schreiner, W., S. Sokolovskiy, D. Hunt, C. Rocken, and Y.-H. Kuo, 2011: Analysis of GPS radio occultation data from the FORMOSAT-3/COSMIC and MetOp/GRAS missions at CDAAC. Atmos. Meas. Tech., 4, 2255-2272, https://doi.org/ 10.5194/amt-4-2255-2011.

Tan, J., G. J. Huffman, D. T. Bolvin, and E. J. Nelkin, 2019: IMERG V06: Changes to the morphing algorithm. J. Atmos. Oceanic Technol., 36, 2471-2482, https://doi.org/10.1175/ JTECH-D-19-0114.1.
Turk, F. J., and Coauthors, 2021: Interpretation of the precipitation structure contained in polarimetric radio occultation profiles using passive microwave satellite observations. J. Atmos. Oceanic Technol., 38, 1727-1745, https://doi.org/10.1175/JTECH-D-210044.1.

Wong, S., E. J. Fetzer, M. Schreier, G. Manipon, E. F. Fishbein, B. H. Kahn, Q. Yue, and F. W. Irion, 2015: Cloud-induced uncertainties in AIRS and ECMWF temperature and specific humidity. J. Geophys. Res. Atmos., 120, 1880-1901, https:// doi.org/10.1002/2014JD022440.

Zou, X., S. Yang, and P. S. Ray, 2012: Impacts of ice clouds on GPS radio occultation measurements. J. Atmos. Sci., 69, 3670-3682, https://doi.org/10.1175/JAS-D-11-0199.1. 